\title{
INDICADORES PARA LA EVALUACIÓN INTEGRAL DE LA PRODUCTIVIDAD ACADÉMICA EN LA EDUCACIÓN SUPERIOR
}

\section{(Indicators for a Comprehensive Evaluation of Academic Productivity in Higher Education)}

\begin{tabular}{|c|c|}
\hline & por \\
\hline$\underline{\text { Article record }}$ & $\begin{array}{l}\text { María Estela Martínez Castro } \\
\text { (estelamartinezmx@yahoo.com.mx) }\end{array}$ \\
\hline$\underline{\text { About authors }}$ & 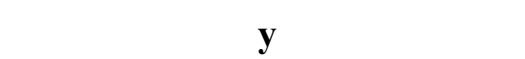 \\
\hline$\underline{\text { HTML format }}$ & $\begin{array}{l}\text { Gerardo Coronado Ramírez } \\
\text { (hinicuil98@yahoo.com) }\end{array}$ \\
\hline
\end{tabular}

Ficha del artículo

$\underline{\text { Sobre los autores }}$

Formato HTML

\begin{abstract}
This article reports the results of an evaluation of academic productivity of three academic departments of the University of Guadalajara, Mexico, through an interrelated system of evaluative indicators it is possible to construct an effective method for identifying the levels and degree of equilibrium in the academic productivity of an institution of higher education.

The method used to evaluate the dimensions of departmental academic productivity permitted interpretation and analysis of the data in order to make comprehensive value judgments and recommendations. Thus, achievements in the areas of research, extension, and teaching were evaluated for the departments observed. The criteria for analyzing the indicator were: effectiveness, efficacy, efficiency, adequacy, and
\end{abstract}

\section{Keywords}

Teaching, Higher Education, Effectiveness, Efficacy, Efficiency, Adequacy, Extension, Research, Indicators, Evaluation of Academic Productivity, Comprehensive Evaluation Departmental Evaluation.

\begin{tabular}{l} 
Resumen \\
El artículo reporta resultados de la evaluación de la pro- \\
ductividad académica practicada a tres departamentos de la \\
Universidad de Guadalajara, en México, mediante la utili- \\
zación de un esquema valorativo e interrelacionado de \\
indicadores con el que se demuestra que es posible cons- \\
truir un método efectivo para identificar los niveles y el \\
grado de equilibrio existente en la productividad académi- \\
ca en una institución de educación superior. \\
La propuesta consiste en un sistema evaluativo que com- \\
bina las principales áreas de actuación universitarias con \\
criterios valorativos, lo que permitió la interpretación y el \\
análisis de los datos para emitir juicios de valor y reco- \\
mendaciones de una forma integrada; esto significa que se \\
consideraron en conjunto los logros correspondientes en \\
las áreas de investigación, extensión y docencia de los \\
departamentos observados, bajo los criterios de efectivi- \\
dad, eficacia, eficiencia, suficiencia y actualización. \\
\hline Descriptores \\
Docencia, Educación superior, Efectividad, Eficacia, Efi- \\
ciencia, Extensión, Evaluación productividad académica, \\
Evaluación integral, Evaluación departamental, Indicado- \\
res, Investigación, Suficiencia
\end{tabular}




\section{Introducción}

La Universidad de Guadalajara ha demostrado su permanente preocupación por adaptarse a las tendencias que van marcando el destino de las universidades públicas de México; también ha manifestado ser una organización educativa que avanza al ritmo que exige el proceso mismo de modernización de su entorno y de la comunidad universitaria. Ello ha sido posible gracias al impulso y fortalecimiento de procesos internos de evaluación y de regulares ejercicios de autoevaluación institucional.

A raíz de su última reforma académica, a principios de los noventa, los esfuerzos por medir sus niveles de calidad se han multiplicado utilizando diversos mecanismos valorativos, evidenciando que las actividades evaluativas se han convertido en una acción regular y un elemento de gran utilidad para la toma de decisiones en la vida de esta institución. La realización de las tareas mencionadas ha permitido avanzar significativamente en el logro de la calidad de los servicios que esta casa de estudios brinda a la comunidad y a sus integrantes, sin embargo se empiezan a observar algunos efectos no deseados en el funcionamiento universitario que pueden asociarse con el sentido que han tomado las acciones evaluativas efectuadas en su interior.

Se puede mencionar, por ejemplo, que los resultados de las evaluaciones institucionales son presentados de manera aislada, sin establecer correlaciones entre las áreas evaluadas, lo que aumenta el riesgo de ofrecer visiones sesgadas, incompletas e inclusive erróneas del funcionamiento universitario.

Ante esta situación, para nada deseable, surgió la inquietud de proponer ejercicios evaluativos que eviten la distorsión analítica, que reduzcan al mínimo procesos de simulación y que no produzcan tensiones infructuosas entre los miembros de la comunidad.

Entonces, se asume como conveniente un sistema de evaluación institucional basado en indicadores que aporten datos significativos sobre aspectos particulares y que, relacionados con otros indicadores, puedan proporcionar una visión integrada de las funciones sustantivas que se realizan en cada una de las instancias operativas de la universidad, particularmente de las académicas.

Mediante la utilización de este sistema integrado de indicadores se podrían obtener resultados de mayor precisión que hiciesen posible sugerir acciones tendientes a mejorar la productividad de un departamento académico; mejoría que no se encontraría referida solamente al aspecto compensatorio o financiero que premie a unos cuantos, sino más bien que detecte aquellos elementos de las actividades académicas sustanciales que requieran ser fortalecidos y aumenten su productividad no en términos de cantidad sino, sobre todo, de calidad, con la intención de que se realicen sus trabajos como lo establece la figura departamental prefigurada en sus documentos normativos y planes de desarrollo; es decir, por núcleos de especialización disciplinar que integren armoniosamente las funciones sustantivas universitarias y no por centros especializados en una actividad académica dispersa.

Darle este rasgo definitorio de integralidad a la evaluación que se realiza regularmente en la Universidad de Guadalajara (UdeG), fue lo que inspiró el interés por recuperar muchos de los datos que existen de los diferentes procesos evaluativos universitarios ya sea mediante el análisis de los reportes de actividades académicas, informes de las rectorías de algunos centros universitarios y de la rectoría general de la misma universidad, así como la opinión de autoridades académicas departamentales, para con todo ello conformar una propuesta instrumental susceptible de ser empleada por los departamentos y que permitiese emitir juicios valorativos de la profundidad, eficacia, efectividad y pertinencia de su acción de forma totalizadora y correlacionada.

El trabajo aquí presentado ofrece los resultados de los esfuerzos realizados para alcanzar este objetivo, proceso que permitió aportar una 
Martínez Castro, M.E. y Coronado Ramírez, Gerardo. (2003). Indicadores para la evaluación integral de la productividad académica en la educación superior. RELIEVE:, v. 9, n. 1, p. 45-72.

http://www.uv.es/RELIEVE/v9n1/RELIEVEv9n1_2.htm

herramienta útil para la evaluación de los departamentos con un enfoque distinto al que prevalece en la mayoría de las universidades e instituciones de educación superior, cuyas evaluaciones se ubican en la perspectiva productivista del trabajo académico exigiendo resultados sin relacionarlos con aquellos factores que en alguna medida intervienen para obtenerlos con una mayor calidad.

\section{La Evaluación en las Instituciones de Educación Superior en México}

Es preciso reconocer que la historia de la evaluación de la educación superior en México lleva ya poco más de tres décadas de desarrollo, ya que desde los años setenta la Asociación Nacional de Universidades e Instituciones de Educación Superior (ANUIES) realizó los primeros ejercicios de autoevaluación en este país para elaborar diagnósticos, pronósticos de demanda y crecimiento de programas específicos de desarrollo institucional (ANUIES, 1997, p. 4).

A raíz de esto ANUIES desarrolló en 1984 un esfuerzo de sistematización de la metodología de evaluación para la educación superior, mediante la utilización de criterios y procedimientos generales en este nivel educativo (Casillas, 1995, p. 41).

El espacio dedicado a los procesos de evaluación se fue abriendo más y como consecuencia de los trabajos realizados, en 1989 la Coordinación Nacional para la Planeación de la Educación Superior (CONPES) aprobó la instalación de la Comisión Nacional de Evaluación de la Educación Superior (CONAEVA), con el propósito de impulsar el proceso de evaluación a nivel nacional mediante la formulación de criterios y directrices generales para proponer políticas y acciones tendientes a mejorar la educación superior.

En esta apertura surgieron las siguientes instancias: en 1990 el Fondo para Modernizar la Educación Superior (FOMES), cuya operación de recursos permitió subsanar en parte los rezagos de la inversión en la infraestructura y equipamiento acumuladas durante la década anterior en las instituciones; en 1991 se establecieron los
Comités Interinstitucionales para la Evaluación de la Educación Superior (CIEES), cuyo principal propósito es la evaluación de la calidad de los programas, especialmente los de formación de profesionales en el nivel de licenciatura $\mathrm{y}$ de las funciones de administración institucional y de difusión de la cultura a través de nueve comités establecidos por áreas de conocimientos, integrados por pares académicos de distintas universidades; por último, en 1992 se constituyó la Federación de Instituciones Particulares Mexicanas de Educación Superior (FIMPES), como una agrupación que desarrolló criterios propios de evaluación institucional para sus agremiados.

Se puede asegurar entonces, que en el nivel de educación superior en México los organismos mencionados han sido las principales instancias con programas empleados para el seguimiento del esfuerzo de la evaluación; y han establecido indicadores de funcionamiento institucional y parámetros que orientan el comportamiento esperado de las instituciones principalmente por el interés de obtener la acreditación y el financiamiento, hecho este que ha marcado las tendencias generales de la evaluación en las instituciones educativas del nivel superior.

Por otra parte, cada institución establece distintos procesos y mecanismos para el ingreso, promoción y permanencia de su personal académico, así como el acceso a estímulos económicos, con lo que se propiciará una mayor participación de los cuerpos colegiados en su diseño, operación y evaluación como lo señala el Programa de Desarrollo Educativo 19952000 (ANUIES, 1997, p. 6)

En la Universidad de Guadalajara, además de los programas de evaluación al personal académico que se mencionan más adelante, se han llevado a cabo diversos procesos de evaluación, de los cuales vale la pena mencionar dos: el programa denominado Evaluar para Mejorar, promovido a finales de 1998 por la Vicerrectoría de la propia universidad, en el que se pretendía evaluar a los departamentos y planes 
Martínez Castro, M.E. y Coronado Ramírez, Gerardo. (2003). Indicadores para la evaluación integral de la productividad académica en la educación superior. RELIEVE:, v. 9, n. 1, p. 45-72.

http://www.uv.es/RELIEVE/v9n1/RELIEVEv9n1_2.htm

de estudio en su primera fase mediante la conducción de los colegios departamentales y los comités consultivos de las coordinaciones de cada plan de estudios; para el año 2000 se habían llevado a cabo algunos talleres y el equipo de trabajo se encontraba en la etapa de definición de los indicadores.

El segundo programa es el que se conoce como Autoestudio, creado en el Plan Institucional de Desarrollo 1995-2000 de la propia universidad, "motivado por las nuevas formas del binomio evaluación-financiamiento, que necesaria e inevitablemente cobrarán vigencia en nuestro país" (UdeG, 1996a, p. 140). Entonces se construyó un modelo basado en estándares considerando las áreas de desarrollo institucional: atención a la demanda estudiantil; distribución de la matrícula; eficiencia de la docencia; programas de docencia; personal académico; investigación; difusión de la cultura y extensión de los servicios; apoyo académico; apoyo administrativo; financiamiento y vinculación. Los resultados de esta evaluación se dieron a conocer en 1998.

\section{La evaluación de los académicos}

En cuanto a la evaluación del personal académico, a partir de 1986 se puso en marcha como uno de los proyectos del Programa de la Carrera Docente del Personal Académico (instrumentado desde 1972), incluyéndolo dentro del Proyecto Nacional de Formación del Personal Académico y que se convertiría en 1993 en un instrumento de la política de la educación superior con la creación del Programa Nacional de Superación del Personal Académico conocido como SUPERA.

Además, el Consejo Nacional para la Ciencia y la Tecnología (CONACYT), el Sistema Nacional de Investigadores (SNI) y algunas fundaciones y empresas que invierten fondos en investigación, son organismos que se han encargado de implementar algunos criterios de evaluación para medir el desempeño de los académicos, ya que para otorgar becas y estímulos solicitan ciertos requisitos que se fueron estableciendo como indicadores para la evaluación de la producción académica.

\section{Los académicos en la Universidad de Guadalajara}

La evaluación del desempeño del personal académico de la UdeG, en lo que se refiere a las actividades de investigación, extensión y docencia inició de una manera sistematizada con el mismo proceso de reforma universitaria desde 1990, año en el que se dieron a conocer los criterios para llevar a cabo una homologación del personal académico mediante la documentación probatoria de las actividades realizadas.

Posteriormente se implementaron diversos programas de deshomologación por medio del otorgamiento de estímulos económicos. Estos programas han implicado un proceso de evaluación importante del desempeño académico al interior de la institución, ya que invadió la esfera de producción individual de los académicos, modificando como lo señala Luis Montaño "significativamente, en la mayoría de los casos, la naturaleza y el contenido del trabajo, así como la organización y el comportamiento de los académicos" (Montaño, 1993, pp.14-15).

Uno de los efectos provocados por este tipo de evaluaciones es la elaboración individualizada de productos académicos, "bajo el discurso de la excelencia en las que se representan las desigualdades intelectuales, culturales y sociales" (Rodríguez, 1999, p. 18).

En el discurso de la excelencia se demanda mayor productividad académica y a su vez, la distribución de recursos también es un factor importante, aunque como dice Eduardo Ibarra:

En la distribución de recursos a la ciencia existe la creencia de que son las ciencias 'duras' las que requieren de fuertes inversiones en infraestructura, mientras las mal llamadas ciencias 'blandas' se bastan a sí mismas sólo con lápiz y papel. (Ibarra, 1998b, p.376). 
Martínez Castro, M.E. y Coronado Ramírez, Gerardo. (2003). Indicadores para la evaluación integral de la productividad académica en la educación superior. RELIEVE:, v. 9, n. 1, p. 45-72.

http://www.uv.es/RELIEVE/v9n1/RELIEVEv9n1_2.htm

\section{Método}

Este estudio se llevó a cabo durante el periodo que comprende de marzo del 2000 a marzo del 2001, y los datos que aquí se incluyen corresponden a la producción académica de tres departamentos de la UdeG reportados durante 1997, 1998 y 1999. El trabajo que se presenta recupera información suficiente para llevar a la práctica un proceso de evaluación de manera integrada.

Con la primer información de tipo documental, basada en lecturas tanto de la UdeG como de los procesos de evaluación prevalecientes en su interior, fue posible plantear el problema que se refiere a la productividad académica dentro del modelo departamental existente en la institución. Derivado de la investigación inicial se constató que no existe evaluación específica sobre la productividad académica a nivel departamental en esa universidad; por lo que se establecieron los objetivos de investigación, se desarrollaron las preguntas que orientarían este estudio, así como la justificación del proyecto y su viabilidad.

\section{Objetivo general}

El objetivo general fue indagar comparativamente cuáles son los elementos decisivos de la productividad académica en tres departamentos de la Universidad de Guadalajara. Por lo que es pertinente precisar que la productividad académica se considera como "la integración de resultados derivados de la producción proporcional del personal académico de un departamento, en sus actividades de investigación, docencia y extensión, en relación con las normas establecidas para la evaluación” (Martínez, 2001, p. 43).

Después de construir el marco teórico, se estableció como trabajo principal una evaluación de tipo descriptiva, comparativa, correlacional y valorativa, lo que ayudó en la definición del modelo que debía adoptarse.

Tomando como principio fundamental que toda evaluación se basa en la aplicación de un modelo, ya que es el modelo el que dirige el enfoque conceptual de los términos utilizados, se tomaron los conceptos básicos y el procedimiento del mo- delo de evaluación comparativa mediante la utilización de indicadores, propuesto por Michael Scriven (1991), por lo que dicho procedimiento se expresa desde la implementación del diseño o plan de evaluación a realizar.

La naturaleza de los datos cuantitativos y cualitativos obtenidos en este estudio, permitió también realizar ejercicios estadísticos que a su vez facilitaron la interpretación y el análisis de los resultados, orientados hacia el enjuiciamiento valorativo del cual surgieron las recomendaciones para la toma de decisiones con la principal finalidad de mejorar la productividad académica.

La estrategia operativa y metodológica se divide en cuatro fases que consisten básicamente en la definición de la situación a evaluar; la recopilación de información y aplicación de los indicadores; la sistematización de datos e interpretación de los resultados; y finalmente, mediante el análisis de la información, la emisión de juicios por parte de los evaluadores para terminar en recomendaciones.

\section{Representatividad de la muestra}

Debido al carácter comparativo de esta evaluación, la representatividad de la muestra elegida se basó principalmente en que los departamentos analizados tuvieran características similares. Considerando que en la Universidad de Guadalajara operan trece centros universitarios, siete regionales y seis temáticos en la zona metropolitana de Guadalajara, se juzgó conveniente incluir en este estudio tres departamentos que pertenecieran a distintos centros universitarios para evitar algunos sesgos o repeticiones sobre todo en la operatividad y la dinámica de la productividad académica.

Se estimó la conveniencia de que los departamentos analizados tuvieran factores esenciales en común, básicamente que reportaran la producción de investigaciones, participación en la docencia y llevar a cabo actividades de extensión y difusión del conocimiento. Se incluyeron dos temáticos metropolitanos (Departa- 
Martínez Castro, M.E. y Coronado Ramírez, Gerardo. (2003). Indicadores para la evaluación integral de la productividad académica en la educación superior. RELIEVE:, v. 9, n. 1, p. 45-72.

http://www.uv.es/RELIEVE/v9n1/RELIEVEv9n1_2.htm

mentos A y B) y uno regional (Departamento C).

Los criterios básicos de integración de la muestra fueron la representatividad departamental, significatividad departamental, cobertura disciplinar y viabilidad de acceso informativo.

\section{Áreas y Criterios de Evaluación}

Las áreas o ámbitos de la evaluación fueron las funciones sustantivas: docencia, investigación y extensión; los criterios de análisis de los indicadores fueron: efectividad, eficacia, eficiencia, suficiencia y actualización.

La efectividad se refiere a la pertinencia o funcionalidad, entendida como la coherencia del proyecto institucional y de sus programas con las necesidades y características del área de influencia de la institución. Se mide el impacto social o institucional.

La eficacia es entendida como criterio de relación o correspondencia entre los fines, objetivos y metas con los logros concretos de ejecución. Se refiere a la coherencia entre las actividades desarrolladas por el departamento y sus académicos para cumplir sus fines y los objetivos de cada uno de sus programas.
La eficiencia es el nivel óptimo de funcionalidad y congruencia para posibilitar la concreción de metas con calidad. Se refiere a la coherencia entre recursos invertidos, esfuerzos desplegados y tiempo empleado para el logro de los objetivos.

La suficiencia, para efectos de este estudio se refiere a la capacidad para cubrir las necesidades de insumos financieros y materiales, así como otros requerimientos planteados para el desarrollo de las actividades académicas.

La actualización es entendida como el nivel de vigencia y renovación de conocimientos disciplinares y pedagógicos, de contenidos programáticos, de interés y de reemplazo.

\section{Indicadores}

Tomando como base la determinación de dichas áreas y criterios, se instrumentó el Sistema de Indicadores por Áreas y Criterios de Evaluación cuyos componentes se desglosan en la Tabla 1, por cada dimensión y criterio analizados.

Tabla 1

\section{Indicadores por Áreas y Criterios de Evaluación}

\section{Para el área de la investigación}

\begin{tabular}{|c|c|c|c|c|}
\hline Efectividad & Eficacia & Eficiencia & Suficiencia & Actualización \\
\hline $\begin{array}{l}\text { 1:Interés de la } \\
\text { sociedad por la } \\
\text { investigación } \\
\text { realizada en el } \\
\text { departamento. } \\
\text { 2:Contribuciones } \\
\text { innovadoras al } \\
\text { campo discipli- } \\
\text { nar. } \\
\text { 3:Distinciones a } \\
\text { la investigación }\end{array}$ & $\begin{array}{l}\text { 4:Evaluación interna } \\
\text { en la investigación } \\
\text { 5:Desarrollo en } \\
\text { equipo de los traba- } \\
\text { jos de investigación }\end{array}$ & $\begin{array}{l}\text { 6: Eficiencia en la obten- } \\
\text { ción del financiamiento } \\
\text { para realizar investigacio- } \\
\text { nes } \\
\text { 7: Equipo de cómputo } \\
\text { adecuado para la realiza- } \\
\text { ción de actividades aca- } \\
\text { démicas } \\
\text { 8:Espacios adecuados } \\
\text { para la realización de } \\
\text { actividades académicas }\end{array}$ & $\begin{array}{l}\text { 9:Financiamiento (interno } \\
\text { y externo) suficiente para } \\
\text { realizar investigaciones } \\
\text { 10:Suficiencia del equipo } \\
\text { de cómputo destinado a la } \\
\text { realización de actividades } \\
\text { académicas } \\
\text { 11:Espacios suficientes } \\
\text { para la realización de } \\
\text { actividades académicas }\end{array}$ & $\begin{array}{l}\text { No se incluyeron } \\
\text { indicadores con } \\
\text { este criterio para } \\
\text { el área de la } \\
\text { investigación }\end{array}$ \\
\hline
\end{tabular}


Martínez Castro, M.E. y Coronado Ramírez, Gerardo. (2003). Indicadores para la evaluación integral de la productividad académica en la educación superior. RELIEVE:, v. 9, n. 1, p. 45-72.

http://www.uv.es/RELIEVE/v9n1/RELIEVEv9n1 2.htm

\section{Para el área de la extensión}

\begin{tabular}{|c|c|c|c|c|}
\hline Efectividad & Eficacia & Eficiencia & Suficiencia & Actualización \\
\hline $\begin{array}{l}\text { No se incluyeron } \\
\text { indicadores con } \\
\text { este criterio para } \\
\text { el área de la } \\
\text { extensión }\end{array}$ & $\begin{array}{l}\text { 12:Membresía a sociedades y } \\
\text { asociaciones científicas vincu- } \\
\text { ladas al campo disciplinar } \\
\text { 13: Desarrollo de actividades } \\
\text { editoriales } \\
\text { 14: Capítulos de libros publi- } \\
\text { cados anualmente } \\
\text { 15: Libros publicados anual- } \\
\text { mente } \\
\text { 16: Trabajos presentados en } \\
\text { reuniones científicas o eventos } \\
\text { académicos especializados }\end{array}$ & $\begin{array}{l}\text { 17: Eficiencia de los } \\
\text { mecanismos de apoyo } \\
\text { para la participación } \\
\text { de académicos en } \\
\text { eventos científicos }\end{array}$ & $\begin{array}{l}\text { 18: Suficiencia de } \\
\text { apoyos otorgados } \\
\text { para la participa- } \\
\text { ción de académi- } \\
\text { cos en eventos } \\
\text { científicos }\end{array}$ & $\begin{array}{l}\text { 19: Suscripciones } \\
\text { vigentes a revistas } \\
\text { científicas }\end{array}$ \\
\hline
\end{tabular}

\section{Para el área de la docencia}

\begin{tabular}{|l|l|l|l|l|}
\hline Efectividad & Eficacia & Eficiencia & Suficiencia & Actualización \\
\hline $\begin{array}{l}\text { 20: Programas } \\
\text { docentes exitosos }\end{array}$ & $\begin{array}{l}\text { 22: Tesis dirigidas } \\
\text { por nivel } \\
\text { material didáctico }\end{array}$ & $\begin{array}{l}\text { No se incluyeron } \\
\text { indicadores con } \\
\text { este criterio para el } \\
\text { área de la docencia }\end{array}$ & $\begin{array}{l}\text { 23: Acervo bibliográ- } \\
\text { fico suficiente }\end{array}$ & $\begin{array}{l}\text { 24: Asistencia a cursos de } \\
\text { actualización docente (pe- } \\
\text { dagógica y didáctica) } \\
\text { 25: Asistencia a cursos de } \\
\text { actualización disciplinar } \\
\text { 26: Diseños o actualizacio- } \\
\text { nes de planes y programas } \\
\text { docentes } \\
\text { 27: Acervo bibliográfico } \\
\text { actualizado }\end{array}$ \\
\hline
\end{tabular}

Se entiende por indicadores aquellos "datos cuya característica principal es la síntesis de un aspecto particular cuantitativo o cualitativo de un objeto de estudio y que correlacionados con otros nos proporciona una visión integrada de una situación a evaluar" (Martínez Castro, 2001, p. 39)

\section{Descripción de Indicadores}

Los indicadores que conforman el sistema propuesto en este trabajo de evaluación, son los siguientes:

\section{AREA: Investigación}

\section{Criterio: Efectividad}

Indicador 1: Interés de la sociedad por la investigación realizada en el departamento.

Descripción: Se refiere a la respuesta que tanto la comunidad del entorno del departamento, co- mo la comunidad científica tienen respecto a las actividades y los avances logrados por el departamento. Habrá proyectos cuya relevancia pueda considerarse significativa en términos de cobertura local, regional, nacional e internacional en distintos niveles.

\section{Variables que participan:}

1.1. Número de proyectos de investigación realizados por el departamento

1.2. Número de notas laudatorias emitidas por la comunidad u organismos públicos o privados, nacionales o extranjeros que se hayan emitido respecto al trabajo del departamento; o número de artículos periodísticos o notas televisivas o radiofónicas en las que se presentaron aspectos de cada uno de los proyectos realizados en el departamento.

1.3. Número de convenios con organismos públicos o privados, para la realización de 
Martínez Castro, M.E. y Coronado Ramírez, Gerardo. (2003). Indicadores para la evaluación integral de la productividad académica en la educación superior. RELIEVE:, v. 9, n. 1, p. 45-72.

http://www.uv.es/RELIEVE/v9n1/RELIEVEv9n1_2.htm

programas o proyectos en los que interviene el personal académico del departamento.

\section{Elementos cualitativos:}

1.4. Valoración del nivel de relevancia de la investigación para la sociedad.

1.5. Ámbitos de relevancia de la investigación en el nivel local o regional, nacional e internacional.

\section{Identificación:}

Número de convenios, solicitudes y notas laudatorias emitidas por la comunidad u organismos públicos o privados, nacionales o extranjeros que se hayan emitido respecto a cada uno de los proyectos de investigación del departamento en los últimos tres años.

\section{Estándar deseable:}

Al menos una nota o solicitud de información respecto a los proyectos emitida por cada año o la existencia al menos un convenio de colaboración en programas o proyectos realizados por personal académico del departamento por año.

Indicador 2: Contribuciones innovadoras al campo disciplinar

Descripción: Habrá de obtenerse el grado de significatividad e innovación que poseen las contribuciones derivadas de la investigación dentro del campo que abarca la disciplina del conocimiento de su departamento.

\section{Variables que participan:}

2.1. Número de proyectos asociados a temáticas consideradas de frontera.

\section{Elementos cualitativos:}

2.2. Contribuciones teóricas o metodológicas al campo disciplinar

2.3. Valoración del nivel de significatividad en la innovación de las contribuciones derivadas de la investigación.

\section{Identificación:}

Existencia y valoración de las contribuciones teóricas o metodológicas significativas que se hayan realizado respecto a cada uno de los proyectos de investigación del departamento en los últimos tres años.

\section{Estándar deseable:}

Al menos contar con dos proyectos vigentes asociados a temáticas de frontera y cuya valoración resulte razonablemente significativa a las contribuciones innovadoras derivadas de los mismos.

\section{Indicador 3: Distinciones a la investigación}

Descripción: Se consideran las distinciones otorgadas por organismos externos, tanto a los investigadores adscritos al departamento como a nivel departamental.

\section{Variables que participan:}

1.1. Número de proyectos de investigación realizados por el departamento

3.1. Número de distinciones otorgadas a la investigación por organismos externos.

\section{Identificación:}

Número de distinciones que se hayan emitido respecto a cada uno de los proyectos de investigación del departamento en los últimos tres años.

\section{Estándar deseable:}

Al menos una distinción otorgada a la investigación que realizan los académicos, por cada departamento en cada año.

\section{Criterio: Eficacia}

\section{Indicador 4: Evaluación interna en la inves- tigación}

Descripción: Acciones de evaluación de las actividades de investigación que se efectúan al interior del departamento con la finalidad de 
Martínez Castro, M.E. y Coronado Ramírez, Gerardo. (2003). Indicadores para la evaluación integral de la productividad académica en la educación superior. RELIEVE:, v. 9, n. 1, p. 45-72.

http://www.uv.es/RELIEVE/v9n1/RELIEVEv9n1_2.htm

mejorar la calidad de los productos de los proyectos de investigaciones que ahí se realizan.

\section{Variables que participan:}

4.1. Número de ejercicios de evaluación realizados en el último año destinados a valorar la actividad investigadora.

4.2. Periodicidad de los ejercicios de evaluación realizados para valorar la actividad investigadora por año.

\section{Identificación:}

Número de ejercicios de evaluación de proyectos realizados por cada departamento en el último año.

\section{Estándar deseable:}

Al menos una acción evaluativa destinada a valorar la actividad investigadora por año.

\section{Indicador 5: Desarrollo en equipo de los traba- jos de investigación.}

Descripción: Se refiere al volumen de actividades de investigación que resultan de trabajos grupales, sean estos disciplinarios o interdisciplinarios. Pueden considerarse equipos de trabajo entre varios investigadores, en combinación con asociados e incluso con el apoyo de asistentes. También se considera trabajo en equipo de investigación cuando se desarrollan proyectos de tipo departamental en los que interviene personal académico de diversas categorías, cuando se organizan comités conjuntos de desarrollo de proyectos, o cuando se forman equipos multidisciplinarios para realizar investigación.

\section{Variables que participan:}

1.1. Número de proyectos de investigación realizados por el departamento

5.1. Número de proyectos diseñados y/o realizados por un equipo de investigación

\section{Elementos cualitativos:}

5.2. Desarrollo de trabajos de investigación en equipo

5.3. Formación de equipos multidisciplinarios para realizar la investigación

\section{Identificación:}

Proporción de proyectos de investigación del departamento desarrollados en equipo en los últimos tres años.

\section{Estándar deseable:}

Al menos un proyecto de investigación desarrollado en equipo por cada departamento en cada año.

\section{Criterio: Eficiencia}

Indicador 6: Eficiencia en la obtención del financiamiento para realizar investigaciones

Descripción: Se refiere al nivel de gestión para la obtención de recursos económicos que se ingresan al departamento de acuerdo a las necesidades financieras de los proyectos que realiza, respecto al tiempo empleado para el logro de dicha obtención de recursos de fuentes financieras internas y externas.

\section{Variables que participan:}

1.1. Número de proyectos de investigación realizados por el departamento

6.1. Número de proyectos de investigación financiados por fuentes internas en los últimos tres años.

6.2. Número de proyectos de investigación financiados por fuentes externas en los últimos tres años

6.3. Fuentes de financiamiento externas del departamento 
Martínez Castro, M.E. y Coronado Ramírez, Gerardo. (2003). Indicadores para la evaluación integral de la productividad académica en la educación superior. RELIEVE:, v. 9, n. 1, p. 45-72.

http://www.uv.es/RELIEVE/v9n1/RELIEVEv9n1_2.htm

\section{Elementos cualitativos:}

6.4. Apreciación del nivel de eficiencia de ambas fuentes de financiamiento respecto a la obtención de recursos financieros de apoyo a la investigación en ambas fuentes, por parte de alguno de los grupos de interés involucrados.

\section{Identificación:}

Proporción de proyectos que han conseguido financiamiento mediante fuentes internas y externas

\section{Estándar deseable:}

Valoración eficiente en términos de tiempo para la obtención de recursos provenientes tanto de fuentes de financiamiento internas como externas en apoyo a la investigación.

\section{Indicador 7: Equipo de cómputo adecuado pa-} ra la realización de actividades académicas

Descripción: Se refiere a las condiciones de adecuación en las que se encuentra el equipo de cómputo en relación con las necesidades planteadas por los proyectos que realiza el departamento.

\section{Variables que participan:}

7.1. Número de colaboradores académicos del departamento.

7.2. Número de equipos de cómputo que tiene el departamento para uso académico

\section{Elementos cualitativos:}

7.3. Valoración respecto a la adecuación del equipo de cómputo que utiliza el personal académico.

\section{Identificación:}

Proporción y valoración del equipo de cómputo utilizado por el personal académico

\section{Estándar deseable:}

Valoración del equipo de cómputo como adecuado para la realización de actividades académicas.

Indicador 8: Espacios adecuados para la realización de actividades académicas

Descripción: Condiciones de los espacios de trabajo que posee el departamento para el desempeño de sus actividades académicas.

\section{Variables que participan:}

8.1. Número de cubículos destinados al trabajo académico

\section{Elementos cualitativos:}

8.2. Valoración de la adecuación de las instalaciones del departamento que son destinadas al trabajo académico.

\section{Identificación:}

Proporción de los espacios destinados al trabajo académico

\section{Estándar deseable:}

Espacios calificados como adecuados para la realización de actividades académicas.

\section{Criterio: Suficiencia}

Indicador 9: Financiamiento (interno y externo) suficiente para realizar investigaciones

Descripción: Esta referido a la cantidad de recursos que se ingresan al departamento de acuerdo a las necesidades financieras de los proyectos que realiza.

\section{Variables que participan:}

6.1. Número de proyectos de investigación financiados por fuentes internas

6.2. Número de proyectos de investigación financiados por fuentes externas 
Martínez Castro, M.E. y Coronado Ramírez, Gerardo. (2003). Indicadores para la evaluación integral de la productividad académica en la educación superior. RELIEVE:, v. 9, n. 1, p. 45-72.

http://www.uv.es/RELIEVE/v9n1/RELIEVEv9n1_2.htm

9.1. Monto de los recursos obtenidos para el financiamiento de los proyectos de investigación provenientes de fuentes internas en los últimos tres años.

9.2. Monto de los recursos obtenidos para el financiamiento de los proyectos de investigación provenientes de fuentes externas en los últimos tres años.

\section{Elementos cualitativos:}

9.3. Valoración del nivel de suficiencia respecto a la obtención de recursos financieros de apoyo a la investigación en ambas fuentes.

\section{Identificación:}

Proporción por proyecto de los recursos que ingresaron para financiamiento de la investigación durante los últimos tres años y nivel de suficiencia de dichos recursos.

\section{Estándar deseable:}

Calificación como suficiente por parte de alguno de los grupos de investigación involucrados.

Indicador 10: Suficiencia del equipo de cómputo destinado a la realización de actividades académicas.

Descripción: Se refiere a la relación equipos/académicos existente para la realización de actividades académicas.

\section{Variables que participan:}

7.1. Número de colaboradores académicos del departamento.

7.2. Número de equipos de cómputo que tiene el departamento para uso académico

\section{Elementos cualitativos:}

10.1. Valoración respecto al nivel de suficiencia del equipo de cómputo utilizado por el personal académico.

\section{Identificación:}

Proporción y valoración de la suficiencia del equipo de cómputo utilizado por el personal académico.

\section{Estándar deseable:}

Al menos un equipo de cómputo por académico cuya valoración se considere como suficiente

Indicador 11: Espacios suficientes para la realización de actividades académicas

Descripción: Nivel de suficiencia de los espacios de trabajo que posee el departamento para el desempeño de sus actividades académicas.

\section{Variables que participan:}

7.1. Número de colaboradores académicos del departamento.

8.1. Número de cubículos destinados al trabajo académico

\section{Identificación:}

Proporción del espacio destinado al trabajo académico.

\section{Estándar deseable:}

Al menos un cubiculo por académico

\section{AREA: Extensión}

\section{Criterio: Eficacia}

Indicador 12: Membresía a sociedades y asociaciones científicas vinculadas al campo disciplinar

Descripción: Se refiere a la cantidad y tipo de adscripción que tiene el departamento (o sus miembros) a sociedades y asociaciones disciplinares. 
Martínez Castro, M.E. y Coronado Ramírez, Gerardo. (2003). Indicadores para la evaluación integral de la productividad académica en la educación superior. RELIEVE:, v. 9, n. 1, p. 45-72.

http://www.uv.es/RELIEVE/v9n1/RELIEVEv9n1_2.htm

\section{Variables que participan:}

7.1. Número de colaboradores académicos del departamento.

12.1. Número de asociaciones a las que se encuentra afiliado el personal académico del departamento.

\section{Identificación:}

Proporción de membresías a sociedades científicas o académicas.

\section{Estándar deseable:}

Al menos una membresía a sociedades científicas o académicas por cada académico del departamento.

Indicador 13: Desarrollo de actividades editoriales

Descripción: Se refiere al volumen y caracterización del destino de las publicaciones periódicas, manuales, reportes técnicos de investigación, o ensayos que se generan por los integrantes del departamento.

\section{Variables que participan:}

7.1. Número de colaboradores académicos del departamento.

13.1. Número de artículos elaborados para difusión científica por cada uno de los académicos del departamento.

13.2. Número de artículos elaborados para divulgación científica por cada uno de los académicos del departamento.

13.3. Número de manuales, reportes técnicos o ensayos de distribución interna elaborados por cada académico del departamento.

\section{Identificación:}

Proporción de actividades editoriales por académico en los últimos tres años

\section{Estándar deseable:}

Al menos la realización de una actividad editorial por académico por año.

\section{Indicador 14: Capítulos de libros publicados anualmente}

Descripción: Es la proporción anual de capítulos de textos o tratados que escriben los académicos adscritos al departamento.

\section{Variables que participan:}

7.1. Número de colaboradores académicos del departamento.

14.1. Número de capítulos elaborados por cada académico

\section{Identificación:}

Proporción de capitulos de libros elaborados por los académicos del departamento en los últimos tres años.

\section{Estándar deseable:}

Al menos la elaboración de un capítulo de libro por año elaborado por cada académico del departamento.

\section{Indicador 15: Libros publicados anualmente}

Descripción: Se refiere a la producción especializada de textos que corresponden al campo disciplinar del departamento que se publican anualmente.

\section{Variables que participan:}

15.1. Número total de libros publicados por el departamento en los últimos tres años

\section{Identificación:}

Promedio de libros publicados por el departamento anualmente 
Martínez Castro, M.E. y Coronado Ramírez, Gerardo. (2003). Indicadores para la evaluación integral de la productividad académica en la educación superior. RELIEVE:, v. 9, n. 1, p. 45-72.

http://www.uv.es/RELIEVE/v9n1/RELIEVEv9n1_2.htm

\section{Estándar deseable:}

Al menos la publicación de un libro por año en cada departamento

Indicador 16: Trabajos presentados en reuniones científicas o eventos académicos especializados.

Descripción: Presentación anual de ponencias y/o conferencia por parte de los integrantes del departamento en eventos especializados de análisis o difusión científica locales o regionales, nacionales e internacionales.

\section{Variables que participan:}

7.1. Número de colaboradores académicos del departamento.

16.1. Número de ponencias y/o conferencias que presentó el personal académico del departamento en foros locales o regionales en los últimos tres años.

16.2. Número de ponencias y/o conferencias que presentó el personal académico del departamento en foros nacionales en los últimos tres años.

16.3. Número de ponencias y/o conferencias que presentó el personal académico del departamento en foros internacionales en los últimos tres años.

\section{Identificación:}

Proporción anual por académico de presentaciones en foros académicos especializados locales, regionales, nacionales e internacionales.

\section{Estándar deseable:}

Al menos una presentación anual en foros académicos ya sea locales, regionales y nacionales y una presentación en foros internacionales, por cada académico.

\section{Criterio: Eficiencia}

Indicador 17: Eficiencia de los mecanismos de apoyo para la participación de académicos en eventos científicos.

Descripción: Se refiere al nivel de gestión para la obtención de apoyos otorgados al personal académico para su participación en eventos científicos.

\section{Variables que participan:}

7.1. Número de colaboradores académicos del departamento.

16.1. Número de ponencias y/o conferencias que presentó el personal académico del departamento en foros locales o regionales en los últimos tres años.

16.2. Número de ponencias y/o conferencias que presentó el personal académico del departamento en foros nacionales en los últimos tres años.

16.3. Número de ponencias y/o conferencias que presentó el personal académico del departamento en foros internacionales en los últimos tres años.

\section{Elementos cualitativos:}

17.1. Valoración en términos de eficiencia para el otorgamiento de recursos de apoyo para la participación académica en eventos científicos.

\section{Identificación:}

Valoración del nivel de eficiencia en la gestión para la obtención de apoyos otorgados al personal académico para su participación en eventos científicos.

\section{Estándar deseable:}

Calificación de la gestión como eficiente para la obtención de recursos de apoyo a la participación de académicos en eventos cientificos. 
Martínez Castro, M.E. y Coronado Ramírez, Gerardo. (2003). Indicadores para la evaluación integral de la productividad académica en la educación superior. RELIEVE:, v. 9, n. 1, p. 45-72.

http://www.uv.es/RELIEVE/v9n1/RELIEVEv9n1_2.htm

\section{Criterio: Suficiencia}

Indicador 18: Suficiencia de apoyos otorgados para la participación de académicos en eventos científicos.

Descripción: Valoración de la suficiencia en términos financieros de los apoyos otorgados al personal académico para su participación en eventos científicos.

\section{Variables que participan:}

7.1. Número de colaboradores académicos del departamento.

16.1. Número de ponencias y/o conferencias que presentó el personal académico del departamento en foros locales o regionales en los últimos tres años.

16.2. Número de ponencias y/o conferencias que presentó el personal académico del departamento en foros nacionales en los últimos tres años.

16.3. Número de ponencias y/o conferencias que presentó el personal académico del departamento en foros internacionales en los últimos tres años.

\section{Elementos cualitativos:}

18.1. Valoración en términos de suficiencia financiera respecto al apoyo otorgado para la participación de académicos en eventos científicos

\section{Identificación:}

Valoración del nivel de suficiencia en la obtención de apoyos financieros otorgados al personal académico para su participación en eventos cientificos.

\section{Estándar deseable:}

Calificación del financiamiento como suficiente para apoyar la participación de académicos en eventos científicos.

\section{Criterio: Actualización}

Indicador 19: Suscripciones vigentes a revistas científicas

Descripción: Se refiere al total de revistas científicas vigentes a las que se encuentra suscrito el departamento, como parte del servicio de actualización de conocimientos del campo disciplinar en los ámbitos nacional e internacional.

\section{Variables que participan:}

19.1. Número total de suscripciones a revistas periódicas que tiene vigentes el departamento

19.2. Número de suscripciones vigentes a nivel nacional

19.3. Número de suscripciones vigentes a nivel internacional

\section{Identificación:}

Vigencia en la suscripción del departamento con revistas cientificas por su origen nacional e internacional.

\section{Estándar deseable:}

Suscripción al menos de una revista cientifica de origen nacional y dos internacionales por departamento.

\section{AREA: Docencia}

\section{Criterio: Efectividad}

\section{Indicador 20: Programas docentes exitosos}

Descripción: Se referirá esta información a la proporción de los planes de estudio que ofrece el departamento y que cuenten por lo menos con una de las siguientes característi$\operatorname{cas}^{[1]}$

$\checkmark \quad$ Alta demanda (número superior en más del 3\% de aspirantes rechazados en solicitudes de ingreso, con relación a la capacidad instalada) 
Martínez Castro, M.E. y Coronado Ramírez, Gerardo. (2003). Indicadores para la evaluación integral de la productividad académica en la educación superior. RELIEVE:, v. 9, n. 1, p. 45-72.

http://www.uv.es/RELIEVE/v9n1/RELIEVEv9n1_2.htm

$\checkmark \quad$ Reconocimiento de instancias oficiales como programa de calidad comprobable

$\checkmark \quad$ Proporción adecuada de titulados respecto a egresados, según la normatividad existente en cada programa docente.

$\checkmark \quad$ Proporción adecuada de egresados trabajando en el área de formación, según la normatividad existente en cada programa docente.

\section{Variables que participan:}

20.1. Número de programas académicos formativos que ofrece el departamento.

20.2. Niveles académicos que atiende el departamento.

20.3. Número de programas que tienen al menos una de las características señaladas.

\section{Identificación:}

Número de programas docentes considerados exitosos

\section{Estándar deseable:}

Ofrecer al menos un programa docente exitoso por departamento.

Indicador 21: Elaboración de material didáctico

Descripción: Es la diversidad y cantidad de material didáctico diseñado y elaborado por los académicos del departamento.

\section{Variables que participan:}

7.1. Número de colaboradores académicos del departamento.

21.1. Número total de materiales didácticos elaborados por académicos del departamento

21.2. Clasificación del material didáctico que se elaboró en el departamento durante 3 años.

\section{Identificación:}

Proporción anual de materiales didácticos elaborados por el departamento.

\section{Estándar deseable:}

Elaboración anual de al menos dos materiales didácticos de cualquier tipo, por académico.

\section{Criterio: Eficacia}

\section{Indicador 22: Tesis dirigidas por nivel}

Descripción: Será la proporción de tesis dirigidas por el personal académico del departamento según los niveles de estudios que estuviesen atendiendo.

\section{Variables que participan:}

7.1. Número de colaboradores académicos del departamento.

22.1. Número total de tesis dirigidas por académicos del departamento en los últimos tres años.

\section{Identificación:}

Proporción anual de tesis dirigidas por el personal académico del departamento $y$ concluidas en los últimos tres años.

\section{Estándar deseable:}

Al menos una tesis dirigida anualmente por cada uno de los integrantes del personal académico del departamento.

\section{Criterio: Suficiencia}

\section{Indicador 23: Acervo bibliográfico suficiente}

Descripción: Se trata de indagar si el personal académico cuenta con el suficiente acervo bibliográfico que necesita para realizar sus actividades de investigación y docencia. 
Martínez Castro, M.E. y Coronado Ramírez, Gerardo. (2003). Indicadores para la evaluación integral de la productividad académica en la educación superior. RELIEVE:, v. 9, n. 1, p. 45-72.

http://www.uv.es/RELIEVE/v9n1/RELIEVEv9n1 2.htm

\section{Variables que participan:}

27.1. Número total de títulos que ofrece el departamento a sus académicos en su centro de información o biblioteca.

\section{Elementos cualitativos:}

23.1. Valoración de la suficiencia del acervo bibliográfico del departamento.

\section{Identificación:}

Nivel de suficiencia del acervo bibliográfico del departamento para la realización de actividades de investigación y docencia.

\section{Estándar deseable:}

Obtener el nivel de "suficiente" en la valoración del acervo bibliográfico del departamento para la realización de actividades de investigación y docencia.

\section{Criterio: Actualización}

Indicador 24: Asistencia a cursos de actualización docente (pedagógica y didáctica)

Descripción: Cantidad promedio de cursos de actualización pedagógica a los que anualmente asiste el personal académico del departamento.

\section{Variables que participan:}

7.1. Número de colaboradores académicos del departamento.

24.1. Número total de cursos de actualización pedagógica que tomaron los académicos del departamento en los últimos tres años

24.2. Títulos de los cursos de actualización pedagógica que tomaron los académicos del departamento en los últimos tres años.

\section{Identificación:}

Promedio anual de cursos de actualización pedagógica que tomaron los académicos del departamento.

\section{Estándar deseable:}

Al menos la asistencia a un curso anual de actualización pedagógica por académico.

Indicador 25: Asistencia a cursos de actualización disciplinar

Descripción: Cantidad promedio de cursos de actualización disciplinar a los que asiste el personal académico durante un año.

\section{Variables que participan:}

7.1. Número de colaboradores académicos del departamento.

25.1. Número total de cursos de actualización disciplinar que tomaron los académicos del departamento en los últimos tres años.

25.2. Títulos de los cursos de actualización que tomaron los académicos del departamento en los últimos tres años.

\section{Identificación:}

Promedio anual de cursos de actualización disciplinar que tomaron los académicos del departamento.

\section{Estándar deseable:}

Al menos la asistencia a un curso anual de actualización disciplinar por académico.

Indicador 26: Diseños o actualizaciones de planes y programas docentes

Descripción: Proporción de planes y programas de estudio que ofrece el departamento, diseñados o actualizados durante el último trienio.

\section{Variables que participan:}

7.1. Número de colaboradores académicos del departamento.

26.1. Número de planes de estudio y programas del departamento que fueron diseñados o modificados por el departamento en el último trienio. 
Martínez Castro, M.E. y Coronado Ramírez, Gerardo. (2003). Indicadores para la evaluación integral de la productividad académica en la educación superior. RELIEVE:, v. 9, n. 1, p. 45-72.

http://www.uv.es/RELIEVE/v9n1/RELIEVEv9n1_2.htm

\section{Identificación:}

Proporción anual de diseños y actualizaciones de planes de estudio

\section{Estándar deseable:}

Al menos una acción de diseño o actualización de un plan o programa de estudios por académico anualmente

\section{Indicador 27: Acervo bibliográfico actualizado}

Descripción: Se trata de indagar si el personal académico cuenta en términos de actualidad con el acervo bibliográfico que necesita para realizar sus actividades de investigación y docencia.

\section{Variables que participan:}

27.1. Número total de títulos que ofrece el departamento a sus académicos en su centro de información o biblioteca.

\section{Elementos cualitativos:}

27.2. Valoración del acervo bibliográfico del departamento en cuanto a su obsolescencia o actualidad.

\section{Identificación:}

Nivel de actualidad del acervo bibliográfico del departamento para la realización de actividades de investigación y docencia.

\section{Estándar deseable:}

Juicio de "actualizado" en la valoración del acervo bibliográfico del departamento

Es importante mencionar que la mayoría de los indicadores propuestos en este sistema de valoración de la productividad académica ya existen, aunque es una práctica común presentar los datos de manera individual, no como sistemas o conjuntos integradores. Por lo que al extraer aquellos datos para ser analizados de manera integrada y comparativa, la interpretación en conjunto cobra sentido y coherencia proporcionando una panorámica distinta.
A cada uno de los indicadores incluidos se le dio un tratamiento que consistió en la revisión, análisis, replanteamiento, pertinencia, prueba de sensibilidad, incorporación de su estándar normativo, y por último, su aplicación, como se explica a continuación:

La revisión se basó en un reconocimiento de criterios e indicadores que se han utilizado en otros programas o proyectos relacionados con la producción de los académicos, en sus diversas dimensiones de evaluación, tal es el caso de ANUIES, SNI, UdeG y CONACYT, en México, mencionados en párrafos anteriores; así como el Proyecto Nadeau (Proyecto de Validación Institucional e Internacional sobre Criterios e Indicadores de Calidad en la Educación Superior, que consiste en la continuación del proyecto pan-canadiense iniciado y dirigido por Nadeau en 1993, que implicó a 80 universidades canadienses y 250 colegios comunitarios de ese país, permitiendo la identificación de 111 criterios generales y 1447 indicadores de calidad y excelencia para estudiantes, profesores, administradores, programas de estudio, servicios institucionales internos y del contexto institucional externo).

El análisis se fundamentó en determinar cuáles de esos indicadores podrían incluirse o descartarse principalmente por la claridad, viabilidad, confiabilidad y validez de cada indicador.

El replanteamiento y la pertinencia estribaron en ir acotando los indicadores en consideración a su pertinencia y significatividad.

Con el listado preliminar de indicadores se realizó una prueba de sensibilidad que consistió en presentar en su conjunto los indicadores a tres académicos con experiencia en las áreas de investigación, extensión y docencia, que pudiesen responder de manera rápida, confiable y sincera sobre la congruencia de dichos indicadores, en el sentido de indagar si la productividad académica departamental podría ser valorada a través de los indicadores propuestos para tal efecto. 
Martínez Castro, M.E. y Coronado Ramírez, Gerardo. (2003). Indicadores para la evaluación integral de la productividad académica en la educación superior. RELIEVE:, v. 9, n. 1, p. 45-72.

http://www.uv.es/RELIEVE/v9n1/RELIEVEv9n1_2.htm

En cuanto a la incorporación de su estándar normativo, se refiere a la descripción del estándar que ayuda al reconocimiento de que la expresión fáctica de los indicadores será considerada como la correcta, completa o tendencialmente satisfactoria para cada uno de ellos (Coronado, 1999, p. 8).

Dicha incorporación es el resultado de un análisis basado en diversos cuestionamientos que se hicieron como parte de este trabajo a un grupo de investigadores de la propia Universidad de Guadalajara con experiencias similares en la producción académica y a partir de ahí se establecieron los mínimos para cada variable. Los estándares incorporados fueron aceptados por los académicos entrevistados, ya que los consideraron como requerimientos mínimos para el desempeño de las actividades de cada área.

Después de pasar la prueba de sensibilidad, los indicadores fueron aplicados, integrando documentos como informes de actividades de cada departamento; informes de actividades presentadas por los rectores de los respectivos centros universitarios; listados del personal académico adscritos a cada departamento; líneas y proyectos de investigación; oferta de cursos; organigramas departamentales; documentos de información general, como folletos o páginas electrónicas de cada departamento, listados del personal promocionado por la UdeG y la página web de la propia universidad.

Como actividad previa al trabajo de campo se elaboró una guía de cuestionario dirigido a los jefes de los tres departamentos que conforman este estudio y cuya información complementó el análisis de los datos cualitativos considerados importantes sobre aspectos que no fueron encontrados documentalmente.

Para sistematizar esta información se utilizó el análisis intra e interdepartamental, esto significa que se establecieron comparaciones al interior de cada departamento y después entre los departamentos.

\section{Resultados}

\section{Caracterización Departamental}

Se realizó un primer acercamiento comparativo (Caracterización Departamental) que permitió conocer con mayor precisión los aspectos académicos (descriptores) más relevantes de cada uno de los departamentos.

Dichos descriptores fueron:

- Planta académica = número de integrantes de la planta académica incluidos los investigadores y técnicos académicos en sus categorías de titulares, asociados y asistentes.

- Grado Académico Promedio (GAP) = se refiere al nivel de estudios terminados del personal académico en promedio por departamento. ${ }^{[2]}$

- Miembros del Sistema Nacional de Investigadores $=$ número de investigadores pertenecientes al SNI

- Promedio de promoción académica = número de niveles ascendidos del personal académico en el Programa de Estímulos Académicos (PROESA) y en el Programa de Actualización Académica (PROACTUAL), en promedio por departamento.

- Personal que obtiene estímulos en los programas académicos de la $\mathrm{UdeG}=$ número de académicos que obtienen estímulos económicos del Programa de Retención y Estímulos a Grupos de Liderazgo Académico (PRYEGLA), del programa de estímulos a docentes y del programa de estímulos a la productividad académica.

- Grupos de Liderazgo = número de grupos pertenecientes al Programa de Retención y Estímulos a Grupos de Liderazgo Académico (PRYEGLA) adscritos al departamento

- Proyectos de investigación realizados entre 1997 y 1999 
Martínez Castro, M.E. y Coronado Ramírez, Gerardo. (2003). Indicadores para la evaluación integral de la productividad académica en la educación superior. RELIEVE:, v. 9, n. 1, p. 45-72.

http://www.uv.es/RELIEVE/v9n1/RELIEVEv9n1_2.htm

- Estructura organizativa departamental = unidades, coordinaciones o laboratorios que forman el departamento. Así como la interacción entre dichas coordinaciones.

- Programas docentes que se ofrecen $=$ en los distintos niveles de licenciatura, maestría y doctorado.

- Actividades de difusión = son eventos que se ofrecen periódicamente por parte del departamento.

Según los resultados de la caracterización departamental, los tres departamentos:

Cuentan con académicos miembros del Sistema Nacional de Investigadores; participan en programas de promoción académica; participan en el Programa de Grupos de Liderazgo Académico; la proporción del personal académico que se beneficia con los programas de estímulos implementados por la propia universidad en estos tres departamentos rebasó el 35\%, dato cercano que coincide con la política de distribución en el ámbito nacional de estímulos que establece un $30 \%$ de beneficiados en las instituciones educativas.

Desarrollan proyectos de investigación individuales y colectivos; la estructura organizativa

\section{Gráfica 1}

Valoración comparativa de las áreas analizadas en los tres departamentos obedece a la propuesta de modelo departamental implementada para la Red, ya que la figura máxima de toma de decisiones al interior de un departamento es el Colegio Departamental, presidida por el jefe de cada departamento y la existencia de unidades, coordinaciones, laboratorios o academias.

En cuanto a sus actividades de difusión y/o extensión los tres departamentos reportan llevar a cabo periódicamente ciertos eventos, como son los seminarios en el ámbito internacional y actividades de apoyo técnico de carácter permanente en el ámbito local y regional.

Planta Académica es el dato utilizado para integrar los resultados de la producción proporcional del personal académico en cada departamento

\section{Aplicación del Sistema de Indicadores}

En la Gráfica 1 se puede observar comparativamente la valoración realizada a los tres departamentos por áreas y con respecto a los estándares mínimos establecidos.

\section{Departamento A}

En términos generales el Departamento A llegó casi a los estándares mínimos establecidos en este estudio para poder detectar su nivel de productividad académica, pues sumando los resultados finales integrados en cada área, obtuvo un total de 228 puntos, cuyo promedio 
Martínez Castro, M.E. y Coronado Ramírez, Gerardo. (2003). Indicadores para la evaluación integral de la productividad académica en la educación superior. RELIEVE:, v. 9, n. 1, p. 45-72.

http://www.uv.es/RELIEVE/v9n1/RELIEVEv9n1_2.htm

corresponde al $93.82 \%$ respecto al promedio general de 243 puntos.

La investigación constituye el área fuerte del departamento, ya que de sus once indicadores, cuatro rebasan los estándares, cuatro más están en el nivel de los estándares y sólo tres indicadores se ubican por debajo de los estándares mínimos establecidos.

En la extensión, de sus ocho indicadores, dos rebasan los estándares, uno está en el nivel de los estándares y cinco se ubican por debajo de los estándares mínimos establecidos para esta área.

Con respecto a la docencia, de sus ocho indicadores, uno rebasa el estándar, tres están en el nivel y cuatro se ubican por debajo de los estándares mínimos establecidos para el área de docencia.

\section{Departamento B}

La productividad académica del Departamento B se puede medir en un $88.88 \%$ considerando los estándares establecidos en este estudio, ya que la suma de los resultados finales que integran cada área fue de 216 puntos, respecto al promedio general de 243 puntos.

De las tres áreas analizadas, la investigación constituye según los resultados de este estudio el área mayormente fortalecida, ya que de sus once indicadores, dos rebasaron los estándares, cinco más se encuentran en el nivel mínimo de los estándares y cuatro indicadores se ubican por debajo de los estándares mínimos establecidos.

La prioridad otorgada a la investigación se relaciona con la información proveniente de la $\mathrm{Ca}-$ racterización Departamental, en la que se establece que el Departamento B cuenta con una planta académica cuyo Grado Académico Promedio es de Pasantes de Maestría, de los cuales el $17 \%$ son miembros del Sistema Nacional de Investigadores.

El posicionamiento de los indicadores de las áreas de extensión y docencia, se ubican de manera similar ya que de los ocho indicadores que conforman cada una de estas áreas, uno rebasó el estándar, uno se encuentra en el nivel mínimo de los estándares y seis indicadores se sitúan por debajo de los estándares mínimos establecidos, en cada una de ellas.

\section{Departamento C}

El Departamento $\mathrm{C}$ demuestra su liderazgo en las actividades de investigación rebasando por dos puntos el mínimo establecido en los estándares que conforman esa área en este estudio. Pero sumando los resultados finales integrados por cada área, obtuvo un total de 175 puntos, cuyo promedio corresponde al $72.01 \%$ respecto al promedio general de 243 puntos.

La investigación constituye el área fuerte del departamento, ya que de sus once indicadores, seis rebasaron los estándares establecidos, tres se sitúan en el nivel mínimo y solamente dos indicadores se ubicaron por debajo de los estándares mínimos planteados. Esta prioridad otorgada a la investigación se relaciona con la información proveniente de la Caracterización Departamental en la que se establece que el Departamento $\mathrm{C}$ cuenta con una planta académica cuyo Grado Académico Promedio es de Pasantes de Maestría, de los cuales el 6\% son miembros del Sistema Nacional de Investigadores.

El posicionamiento de los indicadores de las áreas de extensión y docencia, se ubican de manera similar ya que de los ocho indicadores que conforman cada una de estas áreas, ninguno rebasó el estándar, solamente uno se encuentra en el nivel mínimo de los estándares y siete indicadores se sitúan por debajo de los estándares mínimos planteados, en cada una de ellas.

Para el caso de la docencia, este departamento en comparación con los otros dos, contó con el porcentaje más alto de académicos que reciben estímulos al desempeño docente $23 \%$, lo que podría significar que los niveles correspondientes a esta área deberían ser más altos. 
Martínez Castro, M.E. y Coronado Ramírez, Gerardo. (2003). Indicadores para la evaluación integral de la productividad académica en la educación superior. RELIEVE:, v. 9, n. 1, p. 45-72.

http://www.uv.es/RELIEVE/v9n1/RELIEVEv9n1_2.htm

\section{Integración de resultados por áreas y cri- terios}

\section{Efectividad en la investigación}

Medir el interés de la sociedad por la investigación realizada en el departamento es un proceso bastante complejo, pero al realizar el cruce de indicadores resulta interesante detectar la congruencia entre los mismos. Los tres departamentos demuestran la existencia de convenios con otros organismos, comprueban que se emitieron notas laudatorias por parte de la comunidad, o por parte de organismos públicos o privados, nacionales y extranjeros.

La autovaloración expresada por los jefes de departamento les concede niveles importantes de significación a las investigaciones y las contribuciones que de ellas se derivan, en los ámbitos regionales, nacionales e internacionales.

Además, la existencia de distinciones otorgadas a la investigación por organismos externos a la propia universidad en los tres departamentos es un elemento importante de congruencia con la efectividad de la investigación a nivel departamental.

Por esto con los resultados obtenidos de los indicadores en esta área, queda demostrado que la investigación en los tres departamentos analizados puede considerarse efectiva.

\section{Eficacia de la investigación}

Los ejercicios de evaluación interna de actividades de investigación realizados por lo menos una vez anualmente se asocian con el desarrollo de trabajos de investigación en equipo, ya que dos departamentos manifestaron realizar dichos ejercicios evaluativos, y los tres departamentos cuentan con equipos de investigación. Por lo que se puede afirmar que sí son eficaces las investigaciones que se llevan a cabo en los departamentos analizados.

\section{Eficiencia de la investigación}

Los tres departamentos manifestaron que cuentan con equipo de cómputo y espacios físicos adecuados para realizar las labores académicas. Aunque por otro lado, también coincidieron los tres departamentos en considerar deficiente el nivel de gestión para obtener recursos financieros de apoyo a la investigación.

Con base en lo anterior, se puede entonces afirmar que la eficiencia de la investigación se relaciona con el aspecto de la obtención de recursos financieros, en cuanto a la gestión de los mismos.

\section{Suficiencia de la investigación}

El promedio de apoyos tanto internos como externos para los proyectos de investigación fue considerado insuficiente en dos departamentos. Este tipo de apoyo financiero cubre los requerimientos básicos, como son sustancias y materiales, en ocasiones se considera el pago de honorarios para asistentes, adquisición de bibliografía, y pagos de viáticos en los casos en que se necesita salir de la ciudad o del país con fines de investigación.

De acuerdo con los resultados obtenidos para este criterio, se puede establecer la correlación entre insuficiencia de espacios para la realización de las actividades académicas y la insuficiencia de financiamiento para los proyectos, aunque los tres departamentos cuentan con suficiente equipo de cómputo. Por lo que se puede afirmar que no es suficiente la capacidad de recursos disponibles para la investigación en los departamentos analizados.

En resumen, el área de la investigación según la evaluación realizada e integrando los resultados de los tres departamentos es efectiva y eficaz lo que puede considerarse como los puntos fuertes dentro del área; y los puntos débiles son la deficiencia, así como la insuficiencia de recursos financieros de apoyo a la investigación.

\section{Eficacia en la Extensión}

La membresía a sociedades y asociaciones científicas vinculadas al campo disciplinar implica la presencia del departamento mediante su personal académico en la presentación de 
Martínez Castro, M.E. y Coronado Ramírez, Gerardo. (2003). Indicadores para la evaluación integral de la productividad académica en la educación superior. RELIEVE:, v. 9, n. 1, p. 45-72.

http://www.uv.es/RELIEVE/v9n1/RELIEVEv9n1_2.htm

resultados de trabajos de investigación principalmente. Uno de los departamentos analizados demuestra tener vinculación al campo disciplinar por pertenecer a diversas asociaciones de su competencia profesional.

En cuanto al desarrollo de actividades editoriales que podría ser la elaboración de artículos de difusión científica, artículos de divulgación científica, o manuales, reportes técnicos o ensayos producidos por los académicos del departamento, solamente uno de estos tres departamentos alcanzó el estándar propuesto.

En lo referente a la elaboración de capítulos ninguno de los tres alcanzó el estándar. La producción especializada de libros en cambio, sí rebasó el estándar en dos departamentos. La presentación anual de ponencias y/o conferencias por parte de los académicos en eventos especializados de análisis o difusión científica fue apenas alcanzada por un departamento (que coincidentemente es el que tiene mayor número de miembros inscritos a sociedades científicas), los otros dos quedaron muy por debajo del estándar.

La correlación entre estos indicadores permite lanzar la afirmación de que la extensión en los tres departamentos no es eficaz.

\section{Eficiencia en la extensión}

Solamente uno de los departamentos valora como eficiente el otorgamiento de recursos para la participación de académicos en eventos especializados, se puede afirmar que no hay eficiencia en la extensión de los departamentos analizados.

\section{Suficiencia en la extensión}

Se consideró aquí la capacidad para cubrir las necesidades de insumos financieros planteados para la realización de actividades propias de la extensión. Desafortunadamente los tres departamentos coincidieron en considerar que es insuficiente el apoyo que se otorga a sus académicos para que puedan participar en eventos científicos.

\section{Actualización en la extensión}

Aún cuando uno de los departamentos cuenta con suscripciones a revistas científicas nacionales e internacionales, no se puede afirmar en términos generales que exista actualización en la extensión de los departamentos.

En resumen, se interpreta que el área de la extensión según la evaluación realizada e integrando los resultados de los tres departamentos no es eficaz, no es eficiente, no es suficiente y no cuenta con los elementos de actualización requeridos.

\section{Efectividad en la docencia}

A nivel departamental cobra relevancia ofrecer al menos un programa docente exitoso y en los resultados obtenidos de este análisis dos departamentos cumplieron con el estándar deseable, el tercer departamento tiene planeado ofrecer en el corto plazo un programa de posgrado.

Otro factor importante para detectar la efectividad en la docencia lo constituye la elaboración de materiales didácticos y las proporciones que resultaron de este análisis no llegaron ni al 0.10 cuando se estableció que al menos dicha proporción fuese de 1 material didáctico elaborado por cada académico del departamento.

Es difícil interpretar que por el sólo hecho de contar con programas docentes exitosos se considere efectiva la docencia cuando resultó nula la elaboración de materiales didácticos.

\section{Eficacia en la docencia}

Aunque sólo se considera un indicador para establecer cierta relación de eficacia en la docencia, los tres departamentos que se incluyeron en este estudio obtuvieron un resultado muy por debajo del estándar en la dirección de tesis. 
Martínez Castro, M.E. y Coronado Ramírez, Gerardo. (2003). Indicadores para la evaluación integral de la productividad académica en la educación superior. RELIEVE:, v. 9, n. 1, p. 45-72.

http://www.uv.es/RELIEVE/v9n1/RELIEVEv9n1_2.htm

\section{Actualización en la Docencia}

El personal académico no reporta en sus informes la asistencia mínima esperada a un curso disciplinar y a un curso de actualización docente (pedagógica y didáctica) por año en ninguno de los tres departamentos. Las proporciones resultantes apenas si llegan en uno de los casos al 0.57 en los cursos de actualización disciplinar.

La preparación y actualización de nuestros profesores en cuestiones pedagógicas y didácticas no rebasó siquiera el 0.10 , proporción bastante baja.

En cuanto a la actualización de planes y programas docentes sólo un departamento logró apenas llegar al estándar mínimo de una acción de este tipo.

Y en lo que se refiere al acervo bibliográfico que necesitan o requieren para realizar sus actividades de investigación y docencia se consideró que sí esta actualizado en los tres departamentos.

Considerando que el personal no se actualiza en el ámbito disciplinar ni pedagógico, y que no actualizaron los planes de estudios se interpreta que no hay actualización en la docencia de estos tres departamentos aún cuando cuentan con el acervo bibliográfico actualizado.

\section{Suficiencia en la docencia}

Aun cuando el acervo fue considerado como actual por los tres departamentos, sólo se valoró como suficiente en uno de ellos, precisamente el que cuenta con su propio centro de documentación, por lo que se interpreta que es insuficiente el apoyo a la docencia en este sentido.

En resumen, se interpreta que el área de la docencia según la evaluación realizada e integrando los resultados de los tres departamentos no es efectiva, no es eficaz, no hay actualización en los docentes, no hay suficiencia de apoyos para la docencia.

\section{Conclusiones}

Mediante la aplicación del sistema integrado de indicadores que se propone en este trabajo de evaluación se confirma la posibilidad de valorar así como de establecer comparaciones valorativas de productividad de significación relevante a nivel departamental en una institución de educación superior.

Se confirmó también el hecho de que si los datos obtenidos en cualquier evaluación son vistos aisladamente, su valor no se aprecia con toda claridad, pero al correlacionarlos y analizarlos de manera entrelazada nos permite establecer comparaciones y dimensionar la información resultante desde una panorámica distinta.

Haber demostrado la posibilidad de una evaluación con la modalidad integral resulta relevante en tanto se considera que las universidades podrán seguir impulsando y fortaleciendo los procesos de evaluación internos con un enfoque distinto al que prevalece en algunas instituciones de educación superior, y mediante la detección de los niveles de productividad académica prevalecientes, sería posible conocer los elementos de las funciones sustantivas que requieren ser fortalecidos para aumentar su calidad. Por lo que el sistema puede emplearse para detectar áreas de oportunidad.

En el caso de esta evaluación la situación actual de la productividad académica en los departamentos deja de ser sólo una presuposición y se convierten en hechos verificables y analizables. Sirve este modelo de evaluación comparativa para detectar la producción por áreas y emitir juicios de valor respecto a la interacción de los indicadores y sus variables por cada área y criterio considerado.

Se sugiere analizar más a fondo la conceptualización que se tiene de la productividad académica de tal forma que se considere como la integración de los resultados en relación con las normas establecidas para tal efecto, normas que por sí mismas implican la producción equilibrada de actividades de las tres áreas que con- 
Martínez Castro, M.E. y Coronado Ramírez, Gerardo. (2003). Indicadores para la evaluación integral de la productividad académica en la educación superior. RELIEVE:, v. 9, n. 1, p. 45-72.

http://www.uv.es/RELIEVE/v9n1/RELIEVEv9n1_2.htm

forman las funciones sustantivas universitarias: investigación, extensión y docencia.

Que las universidades logren rebasar el concepto de evaluación en términos sólo de medición, sanción o control de tal forma que en la práctica se proporcionen elementos de mejora en la toma de decisiones, derivados de las evaluaciones que se realizan al personal académico.

Se considera pertinente ahondar la indagación respecto al impacto de los ejercicios de evaluación, probar sistemas de indicadores, impulsar áreas detectadas como deficitarias, incluir indicadores de mayor complejidad y hacer de los indicadores guías de acción.

Se constató que los instrumentos metodológicos utilizados resultaron adecuados para lograr una aproximación a los resultados de la modernización universitaria bajo el discurso de la excelencia académica, vista desde el ángulo de la productividad de su personal académico.

Una característica importante de los indicadores que se proponen es que por sí mismos integran diversas variables que conforman un aspecto importante de la productividad académica, por lo que se puede partir de cada uno de ellos para realizar estudios posteriores más específicos. Cada indicador nos da una ramificación de posibilidades para realizar análisis más profundos. Lo que significa que si se tiene un interés particular por ampliar la información de algunos indicadores, es posible convertirlos en nuevos ámbitos de la evaluación, sin olvidar la consideración de la viabilidad de acceso informativo como un aspecto que bien puede ser un obstáculo de particular relevancia.

\section{Bibliografía}

Casillas Muñoz, Ma. de Lourdes (1995). Los procesos de Planeación y Evaluación. Temas de hoy en la Educación Superior. No. 10. México: ANUIES.

Consejo Nacional para la Ciencia y la Tecnología (1999). Formato para el ingreso de programas docentes al Padrón de Excelencia de CONACYT. México.

Coordinación Nacional para la Planeación de la Educación Superior (1990). "Manual para la Planeación de la Educación Superior". México: SEP-ANUIES.

Coronado Ramírez, Gerardo (1999). Los Indicadores como Guías para la Gestión Administrativa en las Instituciones Educativas. Mimeo. Abril de 1999. México.

Ibarra Colado, Eduardo (1998a). Prólogo: La Universidad y uno mismo. En Eduardo Ibarra Colado (coordinador), La Universidad ante el espejo de la Excelencia. México: Universidad Autónoma Metropolitana. Unidad Iztapalapa. División de Ciencias Sociales y Humanidades. Segunda Edición.

Ibarra Colado, Eduardo (1998b). El nuevo Conacyt y la Evaluación. Rasgos de la política de ciencia y tecnología (1988-1992”. En Eduardo Ibarra Colado (coordinador), La Universidad ante el espejo de la excelencia. Enjuegos organizacionales (pp. 349-392) México: Universidad Autónoma Metropolitana. Unidad Iztapalapa. División de Ciencias Sociales y Humanidades. Segunda Ibaticaiónolado, Eduardo (1998c). Los Hechos de la Modernización. Tesis básicas y primeras acciones (1988-1992". En: Ibarra Colado, Eduardo (coordinador) La Universidad ante el espejo de la excelencia. Enjuegos organizacionales (pp. 393-443). México: Universidad Autónoma Metropolitana. Unidad Iztapalapa. División de Ciencias Sociales y Humanidades. Segunda Edición.

Ibarra Colado, Eduardo. (1999). La universidad en México hoy: ordenamiento institucional, profesionalización académica y reinvención de subjetividades. En Víctor Manuel Rosario Muñoz (coordinador), La Universidad en México retos y transformaciones (pp. 29-56). México: Universidad de Guadalajara. .

Joint Committee on Standards for Educational Evaluation (1981). Standards for evaluations of educational programs, projects, and materials. Nueva York: Mc Graw-Hill.

Llarena de Thierry, Rocío (1992). Impacto de la Planeación en el Desarrollo de la Educación Superior. En Alfredo Fernández y Laura San- 
Martínez Castro, M.E. y Coronado Ramírez, Gerardo. (2003). Indicadores para la evaluación integral de la productividad académica en la educación superior. RELIEVE:, v. 9, n. 1, p. 45-72.

http://www.uv.es/RELIEVE/v9n1/RELIEVEv9n1_2.htm

tini (Eds.), Dos décadas de planeación de Educación Superior (pp. 53-99). México: ANUIES.

Martínez Castro, María Estela (2001). Evaluación integral de la productividad académica en tres departamentos de la Universidad de Guadalajara. Tesis de Maestría en Educación. México: Universidad del Valle de Atemajac.

Martínez Castro, María Estela (1996). Memoria Histórica del Centro de Estudios de la Información y la Comunicación CEIC/Departamento de Estudios de la Comunicación Social DECS. 1986-1996. . Inédito

Martínez Castro, María Estela (1996). Memoria de Investigación del Departamento de Estudios de la Comunicación Social 1986-1996. Inédito

Martínez Rizo, Felipe (1996). La Calidad de la Educación en Aguascalientes. Diseño de un Sistema de Monitoreo. México: Universidad Autónoma de Aguascalientes. Instituto de Educación de Aguascalientes.

Martínez Rizo, Felipe (1998). La planeación y la evaluación de la educación. En Pablo Latapí Sarre (coordinador), Un siglo de educación en México. Biblioteca Mexicana. (I). México: Fondo de Cultura Económica.

Montaño Hirose, Luis (1993). De la Metáfora al Poder. En Eduardo Ibarra Colado (coordinador), La Universidad ante el espejo de la excelencia. Enjuegos organizacionales (pp. 2-17). México: Universidad Autónoma Metropolitana. U. Iztapalapa. División de Ciencias Sociales y Humanidades.

OECD, Organisation for Economic Co-operation and Development (1993). Education at a Glance. OECD Indicators. Paris: Centre for Educational Research and Innovation.

OECD, Organisation for Economic Co-operation and Development (1994). Making Education Count. Developing and Using International Indicators. París: Centre for Educational Research and Innovation.

Rodríguez Romero, M. Mar (1999). Las comunidades discursivas y el cambio educativo. Serie de Actualización Administrativa No. 12. PROCALDES. México: Universidad de Guadalajara.
Ruiz, José María (1999). Cómo hacer una Evaluación de Centros Educativos. Madrid: Narcea Ediciones. Tercera edición.

Scriven, Michael (1991). Evaluation thesaurus. Sage Publications International Educational and Professional Publisher. Fourth Edition.

SNI (1999). Reglamento del Sistema Nacional de Investigadores. Convocatoria de promoción 1999.

Stufflebeam, Daniel L. y Shinkfield Anthony J. (1995). Evaluación sistemática. Guía teórica y práctica. Temas de educación. Barcelona: Paidós/M.E.C.

Topete Barrera, Carlos. (1997). Alternativas de cambio para el futuro desarrollo de la Educación Superior a inicio del siglo XXI. Tesis para obtener el grado de Doctor en Pedagogía. Universidad Nacional Autónoma de México. México, D.F. (Mimeo)

Topete Barrera, Carlos. (s/f). Indicadores y Estrategias para el mejoramiento de la Calidad en la Educación Superior. Conferencia. México: PROCALDES/Universidad de Guadalajara.

Zorrilla Fierro, Margarita Ma. (1995). Valoración de Resultados de la Formación Universitaria. Un Elemento de la Nueva Cultura de la Evaluación en las IES. México: Universidad Autónoma de Aguascalientes/Programa Interinstitucional de Investigaciones sobre Educación Superior. Primera Edición.

\section{Referencias electrónicas}

ANUIES. (1997) La Evaluación y Acreditación de la Educación Superior en México. Revista de la Educación Superior de la ANUIES. n. 101 ene-mz, http://www.anuies.mx/anuies/revsup/index.ht $\underline{\mathrm{ml}}$ (Consultado en abril de 2000)

Díaz Barriga, Angel (1993) La Evaluación Universitaria en el Contexto del Pensamiento Neoliberal. Revista de la Educación Superior de la ANUIES No. 88. Octubre-Diciembre. http://www.anuies.mx/anuies/revsup/index.ht $\underline{\mathrm{ml}}$ (Consultado en febrero de 2000) 
Martínez Castro, M.E. y Coronado Ramírez, Gerardo. (2003). Indicadores para la evaluación integral de la productividad académica en la educación superior. RELIEVE:, v. 9, n. 1, p. 45-72.

http://www.uv.es/RELIEVE/v9n1/RELIEVEv9n1 2.htm

Gago Huguet, Antonio y Ricardo Mercado del Collado (1995). La Evaluación en la Educación Superior Mexicana. Revista de la Educación Superior de la ANUIES No. 96 OctubreDiciembre.

http://www.anuies.mx/anuies/revsup/index.html (Consultado en abril de 2000)

Martínez Rizo, Felipe. (s/f). Es en verdad buena la eficiencia de su institución? En busca de alternativas de evaluación, a propósito de la propuesta de Alexander Astin" Revista de la Educación Superior de la ANUIES, http://www.anuies.mx/anuies/revsup/index.html

(Consultado en enero de 2000)

Mejía Montenegro, Jaime (1994). La Evaluación Cualitativa de la Educación Superior Mexicana, ¿una perspectiva aplazada?. Revista de la Educación Superior de la ANUIES No. 89. EneroMarzo.

México. http://www.anuies.mx/anuies/revsup/index.html (Consultado en febrero de 2000)

Scriven, Michael (April 2000). Product Evaluation Checklist. The Evaluation Center. http://www.wmich.edu/evalctr/checklists/produ ctevaluation.htm (Consultado en mayo de 2000) Scriven, Michael (s/f) Practical Program Evaluation. En: http:// www.wmich.edu (Consultado en mayo de 2000)

Scriven, Michael (June 2000). The Logic and Methodology of Checklists. En: http://www.wmich.edu (Consultado en julio de 2000)

Scriven, Michael (July 2000). Key Evaluation Checklist (KEC). Primarily for pro- ject/program/organization evaluation). En: http://www.wmich.edu (Consultado en julio de 2000)

Stufflebeam, Daniel L. (November 2000). The Ten Commandments, Constitutional Amendments, and Other Evaluation Checklists. Conferencia presentada en Evaluation 2000. Annual Meeting of the American Evaluation Association. Honolulu, Hawaii. November 4, 2000. Disponible en la dirección: www.wmich.edu/evalctr/checklists/checklistp aperAEA.pdf (Consultado en diciembre de 2000)

\section{Notas}

[1] Tomadas de listados que valoran programas, como el Formato para ingreso de programas docentes al Padrón de Excelencia de CONACYT y los Indicadores y Estrategias para el mejoramiento de la Calidad en la Educación Superior de Carlos Topete.

[2] Este indicador existe en la Universidad de Guadalajara desde 1998 en el proceso de Autoestudio que realizó la misma institución de acuerdo a la siguiente fórmula: grado obtenido por el personal académico, cada grado tiene un valor, los valores obtenidos se suman y ese puntaje se promedia entre el número de académicos del departamento. El resultado corresponde a uno de los valores asignados a cada grado y en ese nivel se ubica a cada departamento. Por ejemplo el grado 7 equivale a nivel de maestría o estudiantes de doctorado. Se puede consultar en: Martínez, Estela (2001). P. 151 
Martínez Castro, M.E. y Coronado Ramírez, Gerardo. (2003). Indicadores para la evaluación integral de la productividad académica en la educación superior. RELIEVE:, v. 9, n. 1, p. 45-72.

http://www.uv.es/RELIEVE/v9n1/RELIEVEv9n1_2.htm

\section{ABOUT THE AUTHORS / SOBRE LOS AUTORES}

María Estela Martínez Castro (estelamartinezmx@yahoo.com.mx) Profesora e Investigadora del Centro Universitario de Ciencias Sociales y Humanidades de la Universidad de Guadalajara, adscrita al Departamento de Estudios de la Comunicación Social, en donde coordina la unidad de difusión. Co-autora de "La evaluación del currículum en la educación superior" En: La Gestión Curricular: Significados, prácticas y experiencias del Desarrollo Curricular, libro próximo a publicarse por la UNIVA. Maestra en Educación por la Universidad del Valle de Atemajac.

Gerardo Coronado Ramírez (chinicui198@yahoo.com) Profesor de Talleres y Seminarios de Análisis de Planeación y Evaluación para la Maestría en Educación de la Universidad del Valle de Atemajac en Guadalajara. Participó en el proceso de planeación de la Universidad de Guadalajara. Actualmente es Jefe de la Unidad de Promoción de Educación Continua Abierta y a Distancia de INNOVA, Universidad de Guadalajara.

\section{$\underline{\text { ARTICLE RECORD / FICHA DEL ARTÍCULO }}$}

\begin{tabular}{|c|c|}
\hline $\begin{array}{l}\text { Reference / } \\
\text { Referencia }\end{array}$ & $\begin{array}{l}\text { Martínez Castro, Maria Estela y Coronado Ramírez, Gerardo (2003). Indicadores para la } \\
\text { evaluación integral de la productividad académica en la educación superior. Revista ELectrónica de } \\
\text { Investigación y EValuación Educativa (RELIEVE), v.9, n. 1. } \\
\text { http://www.uv.es/RELIEVE/v9n1/RELIEVEv9n1_2.htm. Consultado en (poner fe- } \\
\text { cha). }\end{array}$ \\
\hline Title / Título & $\begin{array}{l}\text { Indicadores para la evaluación integral de la productividad académica en la educación superior.[ Indica- } \\
\text { tors for a Comprehensive Evaluation of Academic Productivity in Higher Education ] }\end{array}$ \\
\hline Authors / Autores & María Estela Martínez Castro y Gerardo Coronado Ramírez \\
\hline $\begin{array}{l}\text { Review / } \\
\text { Revista }\end{array}$ & Revista ELectrónica de Investigación y EValuación Educativa (RELIEVE), v. 9, n. 1 \\
\hline ISSN & $1134-4032$ \\
\hline $\begin{array}{l}\text { Publication date / } \\
\text { Fecha de publicación }\end{array}$ & 2003 (Reception Date: 2003 January 16; Publication Date: 2003 Febr. 26 ) \\
\hline $\begin{array}{l}\text { Abstract / } \\
\text { Resumen }\end{array}$ & $\begin{array}{l}\text { This article reports the results of an evaluation of academic productivity of three } \\
\text { academic departments of the University of Guadalajara, Mexico, through an interre- } \\
\text { lated system of evaluative indicators it is possible to construct an effective method for } \\
\text { identifying the levels and degree of equilibrium in the academic productivity of an } \\
\text { institution of higher education. } \\
\text { The method used to evaluate the dimensions of departmental academic productivity } \\
\text { permitted interpretation and analysis of the data in order to make comprehensive value } \\
\text { judgments and recommendations. Thus, achievements in the areas of research, exten- } \\
\text { sion, and teaching were evaluated for the departments observed. The criteria for ana- } \\
\text { lyzing the indicator were: effectiveness, efficacy, efficiency, adequacy, and continuing } \\
\text { education. } \\
\text { El artículo reporta resultados de la evaluación de la productividad académica practica- } \\
\text { da a tres departamentos de la Universidad de Guadalajara, en México, mediante la utili- } \\
\text { zación de un esquema valorativo e interrelacionado de indicadores con el que se de- } \\
\text { muestra que es posible construir un método efectivo para identificar los niveles y el } \\
\text { grado de equilibrio existente en la productividad académica en una institución de edu- }\end{array}$ \\
\hline
\end{tabular}


Martínez Castro, M.E. y Coronado Ramírez, Gerardo. (2003). Indicadores para la evaluación integral de la productividad académica en la educación superior. RELIEVE:, v. 9, n. 1, p. 45-72.

http://www.uv.es/RELIEVE/v9n1/RELIEVEv9n1_2.htm

\begin{tabular}{|l|l|}
\hline & $\begin{array}{l}\text { cación superior. } \\
\text { La propuesta consiste en un sistema evaluativo que combina las principales áreas de } \\
\text { actuación universitarias con criterios valorativos, lo que permitió la interpretación y el } \\
\text { análisis de los datos para emitir juicios de valor y recomendaciones de una forma inte- } \\
\text { grada; esto significa que se consideraron en conjunto los logros correspondientes en las } \\
\text { áreas de investigación, extensión y docencia de los departamentos observados, bajo los } \\
\text { criterios de efectividad, eficacia, eficiencia, suficiencia y actualización. }\end{array}$ \\
$\begin{array}{l}\text { Teaching, Higher Education, Effectiveness, Efficacy, Efficiency, Adequacy, Exten- } \\
\text { sion, Research, Indicators, Evaluation of Academic Productivity, Comprehensive } \\
\text { Evaluation Departmental Evaluation. }\end{array}$ \\
\hline $\begin{array}{l}\text { Keywords } \\
\text { Descriptores } \\
\text { ción productividad académica, Evaluación integral, Evaluación departamental, Indi- } \\
\text { cadores, Investigación, Suficiencia. }\end{array}$ \\
\hline $\begin{array}{l}\text { Institution / } \\
\text { Institución }\end{array}$ & Universidad de Guadalajara (México) \\
\hline Publication site / \\
Dirección
\end{tabular}

\title{
Revista ELectrónica de Investigación y $\mathbf{E V}$ aluación Educativa (RELIEVE)
}

\author{
[ ISSN: 1134-4032 ]
}

(C) Copyright 2002, RELIEVE. Reproduction and distribution of this articles it is authorized if the content is no modified and their origin is indicated (RELIEVE Journal, volume, number and electronic address of the document). // (C) Copyright 2002, RELIEVE. Se autoriza la reproducción y distribución de este artículo siempre que no se modifique el contenido y se indique su origen (RELIEVE, volumen, número y dirección electrónica del documento). 